\title{
Apuntes sobre una pedagogía mediada en la pregunta desde ambientes colaborativos digitales
}

Yury Emilio Jaaman Meza ${ }^{1}$

\begin{abstract}
Resumen
Este estudio pretende valorar y destacar la búsqueda de un aprendizaje significativo y el desarrollo de pensamiento crítico y creativo en aulas virtuales desde una metodología de la indagación basados en el uso de la Webquest como herramienta innovadora y alternativa pedagógicadidáctica en los cursos del programa de filosofía. El propósito de esta metodología es incentivar la indagación y la investigación dirigida a través de la web, en donde se propone al estudiante, quien de antemano conoce la rúbrica mediante la cual será evaluado, realizar tareas siguiendo un proceso preestablecido por el docente-tutor, que implica la realización y organización de actividades con la ayuda de unos recursos provistos. De esta forma, se espera el aprovechamiento de la expansión de la Internet y la mayor acogida de los ambientes virtuales de aprendizaje.
\end{abstract}

Palabras clave: aprendizaje, creativo, descubrimiento, investigación, pregunta. 


\title{
Notes on pedagogy mediated through question from digital collaborative environments
}

\begin{abstract}
This study aims to assess and emphasizes the search of a meaningful learning as well as the development of critical and creative thinking in the virtual classroom by using a methodology of inquiry in a digital environment with the use of WebQuest as an innovative tool and as a pedagogical and learning alternative in the philosophy program. The main goal of this methodology is to encourage inquiry and research lead through the web. From there is proposes to the students to do a task by following a process predetermined by the teacher/tutor which involves the execution and organization of activities with the help of provided resources. The students previously know the signature with which will be evaluated. Thus, is expected to take advantage of the Internet expansion and the greater welcome of the virtual learning environments.
\end{abstract}

Key words: Creative, discovery, learning, research, question.

Recibido: 1 de abril de 2013

Aceptado: 5 de junio de 2013

\section{lntroducción}

"Creemos que las condiciones están dadas como nunca para el cambio social, y que la educación será su órgano maestro. Una educación desde la cuna hasta la tumba, inconforme y reflexiva, que nos inspire un nuevo modo de pensar quiénes somos en una sociedad que se quiere a sí misma. Que aproveche al máximo su creatividad inagotable"

Gabriel García Márquez

Desde la cultura griega hasta la actualidad, en la teoría de la educación ha sido una constante considerar que ella contribuye a ampliar el horizonte vital de la persona, a enriquecer su experiencia y a dar sentido a su mundo a través del conocimiento y de la acción (Jaeger, 1957). La educación conduce a que el universo humano, construido sobre la base del saber y del actuar, sea un ámbito 
cada vez más autónomo y con mayor significación. Se dice que la persona altamente educada debe pensar lógicamente, comunicar el pensamiento, formular juicios relevantes y discriminar entre valores.

Si la educación universitaria desarrolla el hábito reflexivo del estudiante, si le incita a aprender a pensar en forma crítica, él o ella, entenderá los límites del saber, apreciará los intereses vinculantes del investigador, captará sus ataduras sociales, percibirá más fácilmente el vínculo entre conocimiento y práctica, conocimiento e historia, conocimiento y verdad. En una palabra, la formación universitaria se proyecta en la formación ética constructiva y engendra no sólo una vida conforme con la verdad, sino también conforme al bien y a la justicia.

Está investigación va dirigida a todos los docentes y discentes de la Universidad Nacional Abierta y a Distancia (UNAD) que busquen mejorar su quehacer pedagógico en la construcción de un aprendizaje y un conocimiento significativo (De la Torre, 1995) y tiene como finalidad valorar y destacar la importancia de la utilización de la WebQuest (en adelante WQ) como herramienta y como alternativa pedagógica y didáctica en el espacio universitario que posibilite la formación y construcción de un conocimiento significativo, fomente la interdisciplinariedad y motive a estudiantes y tutores a la reflexión sobre la necesidad de crear espacios constructores de sentido desde la indagación y búsqueda selectiva de la información.

Si se pregunta: ¿Sería posible pasar de una educación virtual que sólo prepara individuos para cumplir funciones sociales a un proceso de construcción consciente que posibilite la configuración de personas autónomas y protagonistas de su propia formación? ¿Es posible integrar los principios del aprendizaje constructivista, la metodología de aprendizaje por proyectos y la navegación web para desarrollar el currículo que fomente la interdisciplinariedad y motive a estudiantes y tutores a la reflexión sobre la necesidad de crear espacios virtuales constructores de sentido? La respuesta es afirmativa y se denomina WQ.

La importancia de este trabajo radica en realizar un análisis crítico reflexivo que responda a cuál sería la incidencia del uso de la herramienta WQ dentro del aula virtual en la UNAD, ya que este permite el progreso en la habilidad para participar en discusiones que facilitan el desarrollo de destrezas de pensamiento y de razonamiento tales como: comprender las preguntas, dar respuestas, optar por buenas razones, dar sentido lógico, defender una posición, generar pensamiento crítico y creativo, emplear argumentos y búsqueda de criterios que apoyen los propios juicios. 
Apuntes sobre una pedagogía mediada en la pregunta desde ambientes colaborativos digitales. Artículo original producto de

Inicialmente, se establece la importancia de la educación y el aprendizaje para el desarrollo humano mediante los cuales la comunidad conserva y transmite su peculiaridad física y espiritual (Jaeger, 1957: p. 17), destacando teorías y autores relevantes que han aportado al desarrollo educativo.

En el segundo momento se hace un análisis histórico de la herramienta WQ, destacando orígenes, evolución e importancia de la misma y su incidencia en el proceso educativo y los procesos de gestión investigativa y de aprendizaje. Por último, se expone el sentido y función de la WQ en un curso de filosofía y con la prospectiva de aplicación a otros cursos con ambientes virtuales de aprendizaje.

Los proyectos de la educación actual deben estar encaminados a dar una particular atención a los materiales que puedan ser más relevantes para mejorar el desarrollo de los estudiantes respecto al pensamiento, la creatividad y otros procesos mentales superiores, como son la toma de decisiones, la metacognición, la resolución de problemas, el pensamiento crítico y la comunicación con razonamiento.

Desde este proyecto se le dará un énfasis a la creatividad en relación con la innovación. Ha habido muchas definiciones para explicar el concepto de la creatividad. Sternberg (1993) presenta un enfoque global de la creatividad no sólo como una capacidad, sino también como un proceso conformado por tres tipos de inteligencia:

a. La creativa: capacidad para ir más allá de lo dado y engendrar ideas nuevas y diferentes.

b. La analítica: capacidad para analizar y evaluar ideas, resolver problemas y tomar decisiones.

c. La práctica: capacidad para traducir la teoría en la práctica y las teorías abstractas en realizaciones prácticas.

La pretensión será construir un Ambiente Virtual de Aprendizaje con las herramientas de la WQ, donde los estudiantes reconozcan el contraste de los diversos lenguajes disciplinares propiciando el trabajo de autoaprendizaje, y utilicen como medio los recursos de la Internet que conlleve a un aprendizaje significativo inicial y luego potencie la constitución de red de aprendizaje e investigación para que posteriormente se dé una participación activa del estudiante, docentes-tutores y comunidad al propiciar un ambiente adecuado para el logro de los objetivos educativos a través de una metodología didáctica, que ayude a crear red investigativa. 


\section{Metodología}

Este estudio se desarrolló en un análisis crítico reflexivo con parámetros hermenéuticos del quehacer educativo desde el modelo de la WQ en su concepción, diseño y contextualización al modelo pedagógico de la UNAD, que pretende valorar y destacar la importancia del método y su puesta en práctica en los cursos y en las aulas virtuales con la pretensión de incentivar la investigación guiada como alternativa pedagógica en el aprendizaje que posibilita la formación y construcción de un aprendizaje y conocimiento significativo que atienda a los objetivos de formación.

Desde la puesta en práctica en las aulas virtuales, se aspira también a motivar una experiencia constructiva en la que los docentes-tutores establecen la tarea en función de sus objetivos y los recursos disponibles en la web. Refuerza la idea de que el docente-tutor es quien planifica la actividad para que los estudiantes puedan llevarla a cabo de la forma más autónoma posible, a través de la utilización de los recursos eficazmente y construyendo su propia versión del conocimiento. Por su parte, los estudiantes amplían su autonomía tanto en el espacio como en el tiempo (Bárbara, 2003) y se sienten protagonistas llevando a cabo una investigación para ellos y para el grupo, lo que a su vez los hace actuar con más responsabilidad.

En el siguiente esquema se refleja todo lo expuesto en este apartado acerca del diseño de una WQ. Así, se incluyen los factores a tener en cuenta, los pasos a dar y la actuación en cada uno de ellos en la propuesta de innovación para los cursos.

Tabla 1. Pasos en el diseño práctico de una WQ en cursos académicos en la UNAD

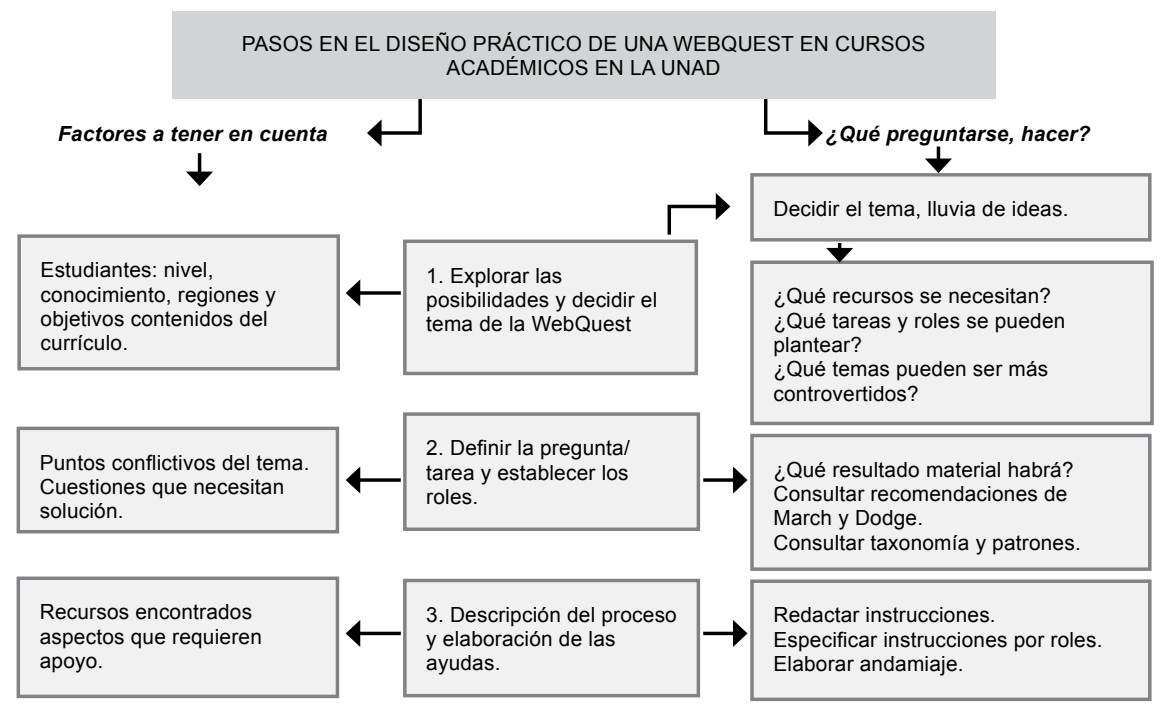

Fuente: Construcción personal del autor de artículo 


\section{Resultados}

La WQ es la aplicación de una estrategia de aprendizaje por descubrimiento guiado a un proceso de trabajo desarrollado por los estudiantes utilizando los recursos de la Internet. WQ significa indagación e investigación a través de la web. Originariamente fue formulado a mediados de 90 hasta los años 2000 por Bernie Dodge (1995; 2000; 2004) en la Universidad de San Diego.

La idea inicial con la que se creó la metodología de trabajo, basada en el objetivo claro de aprender a seleccionar y recuperar datos de múltiples fuentes fue desarrollar las habilidades de pensamiento crítico (Dodge, 2000). Por ello, una WQ consiste, básicamente, en presentarle al estudiante un problema con un conjunto de recursos preestablecidos por el autor de la misma, que suele ser su profesor, de modo que evite la navegación simple y sin rumbo del estudiante a través de la internet (Watson, 1999). Dodge y Muñoz (1997) resumen las conclusiones del proyecto en los siguientes principios que, sin duda, influyeron en el modelo de la WQ que fue diseñado por Dodge poco después de concluir el San Diego Microworld Project:

-Un proyecto constructivista tiene más posibilidades de éxito si incorpora un cierto grado de estructura.

- Ofrecer la oportunidad de adoptar roles específicos normalmente beneficia a aquellos estudiantes que no suelen tener éxito en un entorno de clase tradicional.

-En un proyecto que invita a la creatividad y que permite un final abierto a múltiples posibilidades, es importante establecer límites realistas que faciliten que el proyecto se lleve a cabo sin extenderse demasiado en detalles poco relevantes.

-El tiempo es muy valioso y, por tanto, a la hora de diseñar unidades constructivistas, es importante minimizar el tiempo que se emplea en aquellas tareas que no contribuyen al aprendizaje.

-Para que una innovación se institucionalice es preciso que los creadores del proyecto la compartan deliberadamente a fin de que el mayor número de educadores se sientan partícipes y colaboren.

-WQ es un modelo de aprendizaje extremamente simple y rico para propiciar el uso educativo de Internet, basado en el aprendizaje cooperativo y en procesos de investigación para aprender.

-Una WQ es una actividad enfocada hacia la investigación, en la que la información usada por los estudiantes es, en su mayor parte, descargada de Internet. Básicamente es una exploración dirigida, que culmina con la producción de una página Web, donde se publica el resultado de una investigación. 
-WQ es una metodología de aprendizaje basado fundamentalmente en los recursos que proporciona Internet que incitan a los estudiantes a investigar, potencian el pensamiento crítico, la creatividad y la toma de decisiones, contribuyen a desarrollar diferentes capacidades llevando así a los estudiantes a transformar los conocimientos adquiridos.

Existe en la actualidad mucha información disponible en Internet, destinada a docentes, explicando el concepto, características, proceso de creación y aplicación de las WQ, así como ejemplos ya creados por los propios profesores. La mayor parte de esta información está en inglés, aunque ya empieza a existir algunos documentos y unidades didácticas en español.

La realización de una WQ consiste básicamente en que el docente/tutor identifica y plantea un tópico-problema y a partir de ahí crea una web en la que presenta la tarea al estudiantado, le describe los pasos o actividades que debe que realizar, les proporciona los recursos On line necesarios para que los estudiantes por sí mismos desarrollen ese tópico, así como los criterios con los que serán evaluados. Una WQ, según B. Dodge y T. March (Dodge \& March, s.f.), se compone de seis partes esenciales: introducción, tarea, proceso, recursos, evaluación y conclusión.

Tabla 2. Elementos de una W.Q.

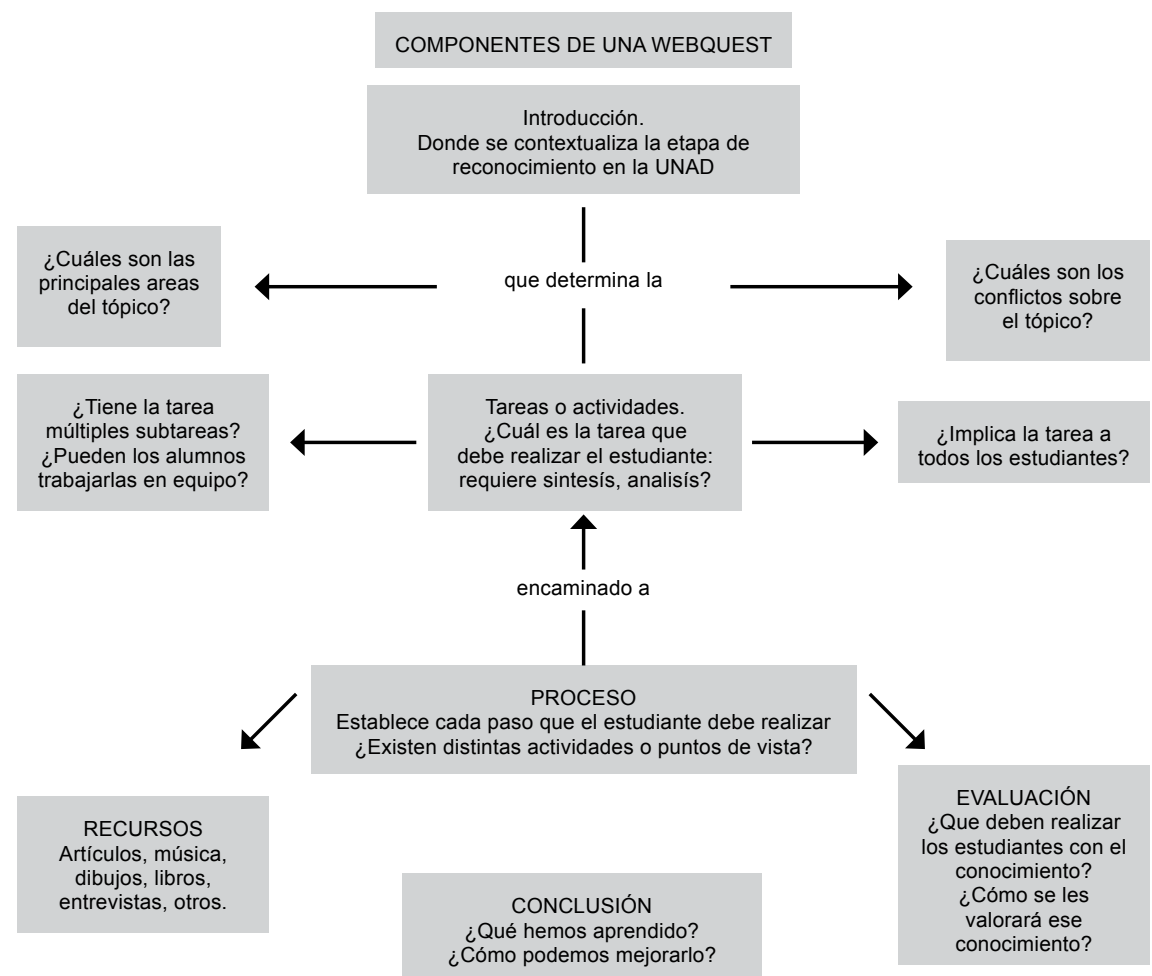

Fuente: Construcción personal del autor de artículo 
La introducción provee a los estudiantes la información y orientaciones necesarias sobre el tema o problema sobre el que debe trabajar. La meta de la introducción es hacer la actividad atractiva y divertida para los estudiantes de tal manera que los motive y mantenga este interés a lo largo de la actividad. Los proyectos deben contarse a los estudiantes haciendo que los temas sean atractivos, visualmente interesantes, parezcan relevantes para ellos debido a sus experiencias pasadas o metas futuras, importantes por sus implicaciones globales, porque necesitan una pronta solución o divertidos ya que ellos pueden desempeñar un papel o realizar algo.

La tarea es una descripción formal de algo realizable e interesante que los estudiantes deberán haber llevado a cabo al final de la WQ. Esto podría ser un producto tal como una presentación multimedia, una exposición verbal, una cinta de video, construir una página web o realizar una obra de teatro. Una WQ exitosa se puede utilizar varias veces, bien en espacios diferentes. Cada vez la actividad puede ser modificada o redefinida y se puede desafiar a los estudiantes para que propongan algo que vaya más lejos, de tal manera, que sea más profunda que las anteriores.

La tarea es la parte más importante de una WQ y existen muchas maneras de asignarla. Para ello, puede verse la taxonomías de tareas (Dodge, 2002c) en la que se describen los 12 tipos de tareas más comunes y se sugieren algunas formas para optimizar su utilización; estas son: Tarea de auto reconocimiento, de producción creativa, científica, de juicio, analíticas, de misterio, de recopilación, de repetición, de persuasión, de construcción de consenso, de diseño y las periodísticas.

El proceso describe los pasos que el estudiante debe seguir para llevar a cabo la Tarea, con los enlaces incluidos en cada paso. Esto puede contemplar estrategias para dividir las Tareas en Subtareas y describir los papeles a ser representados o las perspectivas que debe tomar cada estudiante. La descripción del proceso debe ser relativamente corta y clara.

Para concretar mejor el tipo de procesos cognitivos que los estudiantes pueden llevar a cabo en una WQ, Dodge (2004) se apoya en el modelo de dimensiones de pensamiento y aprendizaje de Marzano (1992). Según este modelo, existen cinco categorías de pensamiento que se corresponden con las "cinco dimensiones del aprendizaje" a saber: dimensión de actitudes y percepciones, de adquirir e integrar el conocimiento, de extender y refinar el conocimiento, de usar significativamente el conocimiento $\mathrm{y}$, finalmente, de hábitos productivos de pensamiento.

Estas cinco dimensiones del aprendizaje no se encuentran aisladas, sino que se relacionan entre sí como se ve en el esquema tomado de Marzano y Pickering (1997). 
Los recursos consisten en una lista de sitios web que el profesor ha localizado para ayudarl a completar la tarea. Estos son seleccionados previamente para que el estudiante pueda enfocar su atención en el tema, en lugar de navegar a la deriva. No necesariamente todos los recursos deben estar en Internet y la mayoría de las WQ más recientes incluyen los recursos en la sección correspondiente al proceso. Con frecuencia, tiene sentido dividir el listado de recursos para que algunos sean examinados por todo el grupo, mientras que otros recursos corresponden a los subgrupos de estudiantes que representarán un papel específico o tomarán una perspectiva en particular. El apartado de recursos aparece integrado dentro del módulo de proceso, de manera que los enlaces y otros recursos se incluyen asociados a cada uno de los roles que suelen asignarse a los estudiantes. Esta inclusión del apartado de recursos dentro del proceso -vinculándolos a las actividades en las que van a necesitarsupone un refuerzo instructivo de la WQ (Novelito, 2004).

Algunos modelos para realizar la WQ proponen en este punto la dirección o guía que el docente pueda brindar a sus estudiantes para explicarles la forma de administrar su tiempo a lo largo del desarrollo de la tarea. Mediante la construcción de ayudas visuales como mapas conceptuales o diagramas que sirvan como bitácora, se muestra al estudiante la forma de conducir la realización de la tarea.

Para guiar en la creación de una WQ de calidad, March (1998d) ha elaborado una hoja de evaluación del diseño de la WQ y una plantilla que nos ayuda a distinguir si cada una de las partes de la WQ está bien definida y se corresponde con el modelo. Los aspectos que se evalúan en dicha hoja de evaluación son (March, 1998d): un comienzo atractivo, la calidad de la pregunta-tarea, la base de conocimiento común para todo el grupo, definición de los roles, el uso de la Web, la transformación del pensamiento que se produce, la retroalimentación del mundo real y la conclusión de la WQ.

En el apartado de evaluación se describe lo más concreta y claramente posible los criterios que se van a aplicar para evaluar el rendimiento de los estudiantes, de acuerdo con los resultados que se anticiparon en la tarea. Se especificará si habrá una calificación común por grupo o calificaciones individuales. Normalmente, junto con la explicación de cuáles son los aspectos que se evaluarán y de qué modo, se incluyen los parámetros exactos que se emplearán para dicha evaluación y se les asigna valores en escala dependiendo del grado de cumplimiento de los objetivos. Esto es lo que en inglés se denomina una rubric, que es el método de evaluación que se utiliza con más frecuencia en la WQ. Dicho término puede ser traducido al español como matriz de valoración.

La matriz de valoración es una herramienta que se considera especialmente útil en los casos en que los criterios de evaluación son complejos y subjetivos Dodge y Pickett (2001), Dodge et al. (2001b), ya que permite separar los 
diferentes aspectos por evaluar en varios componentes, y eso ayuda a decidir su valor más objetivamente. Una evaluación de este tipo, además de ser un instrumento de valoración del rendimiento final de los estudiantes, también es un medio por el que se informa a los estudiantes de lo que deben conseguir y en qué grado. Una de las instrucciones que se suelen dar a los estudiantes en algún momento del proceso es que revisen la matriz de valoración. De esta forma, les sirve de guía y se hacen más conscientes de los objetivos de la WQ, pudiendo comprobar si están cumpliendo lo previsto o no. De hecho algunos autores consideran la matriz de valoración otra parte del andamiaje de la WQ (Hanson, 2001). Este tipo de evaluación es lo que se denomina "evaluación auténtica" porque no utiliza pruebas estandarizadas, sino que mide la actuación de los estudiantes, como cuando se hace una evaluación de cualquier cosa en el mundo real: "The instructor observes the student in the process of working on something real, provides feedback, monitors the student's use of the feedback, and adjusts instruction and evaluation accordingly" (Dodge y Pickett, 2001:1).

Basándonos en las conclusiones de varios autores, a continuación se resumirán las ventajas más significativas del uso de matrices de valoración para evaluar una WQ: permite que la evaluación sea más objetiva y consistente, obliga al profesor a clarificar sus criterios específicamente, así como a comunicar sus expectativas, proporciona retroalimentación acerca de la eficacia de la instrucción, ayuda al estudiante a comprender las cualidades que una determinada tarea debe poseer, muestra al estudiante lo que se espera de él y cómo será evaluado, además permite que el estudiante se autoevalúe.

Por último, en la conclusión se resume lo que los estudiantes han adquirido al completar la WQ, a modo de autorreflexión cuyo objetivo es reforzar lo aprendido. A menudo se les anima a continuar con la investigación posteriormente, incluyendo enlaces o referencias a otro tipo de recursos en la web, bibliográficos, etc.

Quizás lo más interesante de este modelo o estrategia es que el profesorado puede asumir sin grandes costos económicos ni de recursos ni de tiempo, la generación de materiales de aprendizaje destinados a sus estudiantes utilizando la información y servicios disponibles en la internet. Las WQ no requieren la utilización de software complejo ni especializado de creación de programas multimedia. Para que un docente o grupo de profesores estén en condiciones de crear una WQ necesita simplemente tener los siguientes conocimientos y habilidades: saber navegar por la WWW, manejar adecuadamente los motores de búsqueda de información, dominar el contenido o materia que se enseña, y conocimientos básicos del diseño HTML para la creación de documentos hipertextuales (Dodge, 2001a). 


\section{Discusión}

Si se llega al consenso general de que la educación es el principio mediante el cual la comunidad humana conserva y trasmite sus peculiaridades físicas y espirituales y además que posibilita reconstruir el sentido desde un aprendizaje y conocimiento significativo, entonces, también se estará de acuerdo con que el hombre sólo podrá propagar y conservar su forma de existencia social y espiritual mediante las fuerzas por las cuales la ha creado. Es decir, mediante el ejercicio de una voluntad consciente y una razón indagadora, independientemente de los espacios (semipresenciales y/o digitales).

La aplicación de la WQ como herramienta y modelo de desarrollo investigativo en los curso académico de filosofía ha posibilitado respuestas muy positivas en los estudiantes en la UNAD dada la estrategia instructiva diseñada especialmente para la web que más se ha extendido entre la comunidad educativa desde la aparición de internet.

Se puede dictaminar que son dos los factores que han influido en esta acogida de la WQ: en primer lugar, la importancia que la internet ha cobrado en el mundo educativo y, en segundo lugar, la puesta en práctica de uno de los principios que se alcanzaron al final del San Diego Microworld Project, según el cual, para que una innovación se institucionalice es preciso que los creadores del proyecto la compartan deliberadamente con el fin de que el mayor número de educadores se sientan partícipes y colaboren (Dodge y Muñoz, 1997). Desde el primer momento en que la red@gora ha propuesto el modelo WQ. Son muchos los docentes y tutores interesados en integrarse a la propuesta innovadora y la pretensión es difundir el modelo en talleres y cursos para profesores desde la difusión de red:

http://agora-virtual.blogspot.com/, http://www.yuryjaaman.webs.com/, http://comunicandonos.webs.com/introduccion.htm y otras. 


\section{Referencias bibliográficas}

Bárbara, C. (2003). WQ. Una investigación guiada con recurso internet Comunicación presentada en el III Congreso internacional virtual de educación CIVE. Universidad de las islas Beleares, 1-11 de abril de 2003.

De la torre, S. (1995). Evaluación de la creatividad. Madrid: Trillas editores.

Dodge, B. (1995a). Origen del Concepto WQ. Comunitat Catalana de WQ. Recuperado de: http://www.xtec.cat/recursos/W.Q.s/index.htm

Dodge, B. (2000) Thinking Visually with W.Q.s. San diego: San diego State University, Extraído el 30 de julio, 2012 en: http://edweb.sdsu.edu/W.Q./tv/\#

Dodge, B. (2001a). «FOCUS. Five Rules for Writing a Great W.Q.». Learning \& Leading with Technology, vol. 28, No. 8, p. 6-10. Recuperado de: http://www.eduteka.org/ W.Q.Lineamientos.php

Dodge, B. (2001b). Rubric for Evaluating W.Q. s. San Diego State University, Recuperado de: http://W.Q..sdsu.edu/rubrics/weblessons.htm

Dodge, B. (2002a). Adapting and Enhancing Existing $W Q$ s. San Diego State University, Recuperado de: http://W.Q..sdsu.edu/adapting/

Dodge, B. (2002b)WQ Design Patterns. San Diego State University, Recuperado de http://W.Q..sdsu.edu/designpatterns/all.htm

Dodge, B. (2002C) W.Q. taskonomy: taxonomy of tasks. San diego State University, Recuperado de http://W.Q..sdsu.edu/taskonomy.html

Dodge, B. (2004). The W.Q. Design Process. San Diego State University, Recuperado de http://W.Q...sdsu.edu/designsteps/index.html

Dodge, B. y Pickett, N. (2001). "Rubrics for lessons" Recuperado de http://W.Q..sdsu. edu/rubrics/weblessons.html

Dodge y March (s.f), Las WQ y el Uso de la Información. Recuperado de http://www. eduteka.org/comenedit.php3?ComEdID=0010

Dodge y Muñoz (1997). Lessons learned from the San Diego Microworld Project. Recuperado de http://edweb.sdsu.edu/people/bdodge/Bertelsmann.html

Hanson, K. (2001). Beyond read and recall: an introductions to web-based learning using $W Q$. Recuperado de http://connections.smsd.org/highlands/creating_W.Q.s.htm 


\section{Revista de}

Jaeger, W. (1957). Paideia. México: Fondo de Cultura Económica editores.

March, T. (1998d). WQ rubric. Recuperado de http://www.ozline.com/W.Q./rubric. help.html

Marzano, R. J.(1992). A different kind of classroom. Teaching with dimensions of learning. Alexandria, VA: ASCD.

Marzano, R. J. \& Pickering (1997). Dimensions of learning teachers manual. Alexandria, VA: ASCD.

Novelino, B. (2004). El alma de la WQ. Quaderns digitals. Recuperado de http:www. quadernsdigitals.net/index.php?accionMenu=hemeroteca.visualizaArticulolUvisualiza \&articulo_id=7360

Sternberg, R J. (1993). La naturaleza de la creatividad. Nueva York: Cambridge University Press.

Watson, K. L. (1999). W.Q. the middle school curriculum: Promoting technological literacy in the classroom. Recuperado de http://www.ncsu.edu/meridian/jul99/W.Q./ 


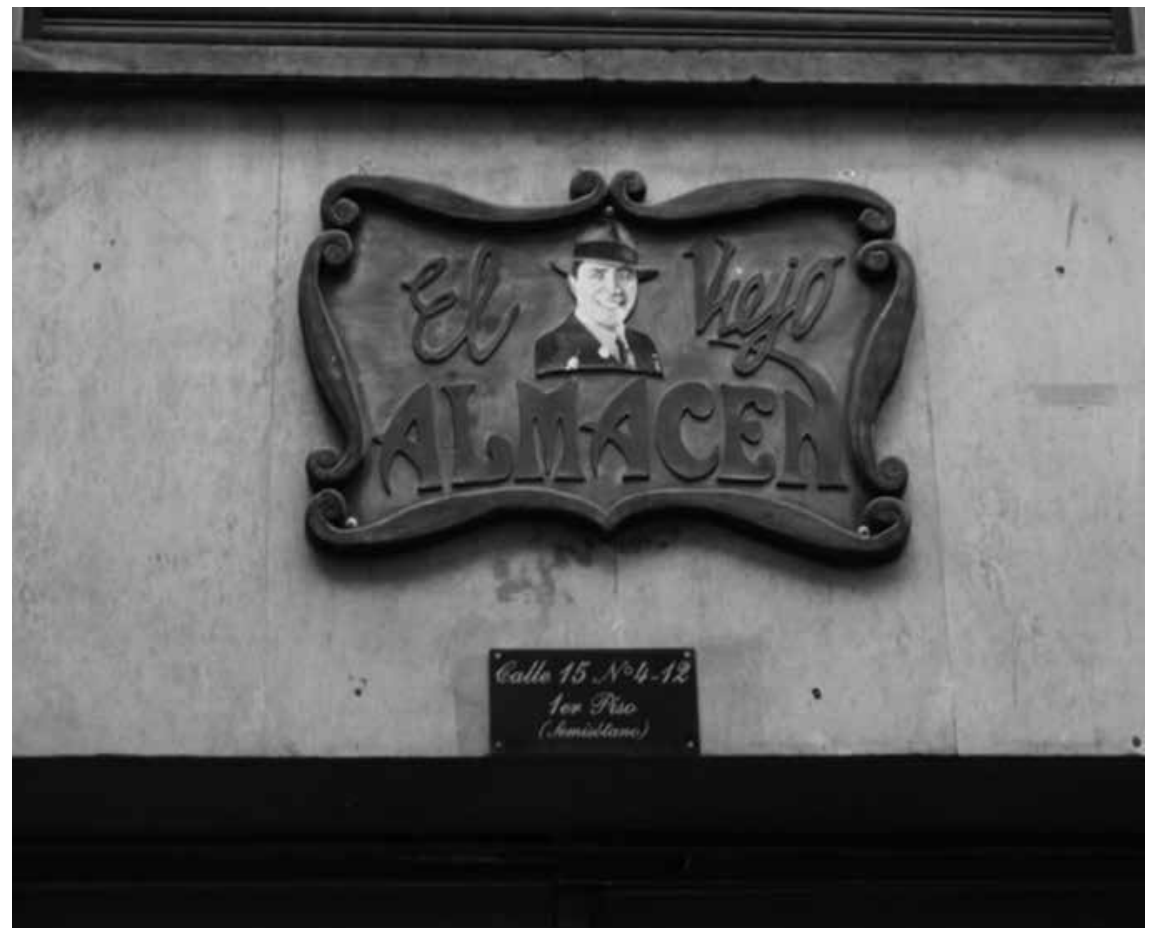

Fotografia: José Enrique Castillo 